\title{
A Prospective, Comparative Study of Ventilation-Perfusion Planar Imaging and Ventilation-Perfusion SPECT for Chronic Thromboembolic Pulmonary Hypertension
}

\author{
Lei Wang ${ }^{1}$, Meng Wang ${ }^{1}$, Tao Yang ${ }^{2}$, Dayong $\mathrm{Wu}^{1}$, Changming Xiong*2, and Wei Fang*1 \\ ${ }^{1}$ Department of Nuclear Medicine, Fuwai Hospital, National Centre for Cardiovascular Diseases, Chinese Academy of Medical \\ Sciences, Beijing, China; and ${ }^{2}$ Centre for Diagnosis and Management of Pulmonary Vascular Diseases, Department of Cardiology, \\ Fuwai Hospital, National Centre for Cardiovascular Diseases, Chinese Academy of Medical Sciences, Beijing, China
}

\begin{abstract}
This study compared the diagnostic performance of ventilation-perfusion (V/Q) planar imaging and V/Q SPECT and determined whether combining perfusion SPECT with low-dose CT (Q-LDCT) may be equally effective in a prospective study of patients with chronic thromboembolic pulmonary hypertension (CTEPH). V/Q scanning is recommended for excluding CTEPH during the diagnosis of pulmonary hypertension $(\mathrm{PH})$. However, V/Q planar imaging and V/Q SPECT have yet to be compared in patients with CTEPH. Methods: Patients with suspected $\mathrm{PH}$ were eligible for the study. $\mathrm{PH}$ attributable to left heart disease or lung disease was excluded, and patients whose $\mathrm{PH}$ was confirmed by right heart catheterization and who completed V/Q planar imaging, V/Q SPECT, Q-LDCT, and pulmonary angiography were included. V/Q images were interpreted and patients were diagnosed as instructed by the 2009 European Association of Nuclear Medicine guidelines, and pulmonary angiography analyses were used as a reference standard. Results: A total of 208 patients completed the study, including 69 with CTEPH confirmed by pulmonary angiography. V/Q planar imaging, V/Q SPECT, and Q-LDCT were all highly effective for diagnosing $\mathrm{CTEPH}$, with no significant differences in sensitivity or specificity observed among the 3 techniques (respective sensitivity and specificity: $94.20 \%$ and $92.81 \%$ for $\mathrm{V} / \mathrm{Q}$ planar imaging, $97.10 \%$ and $91.37 \%$ for $V / Q$ SPECT, and $95.65 \%$ and $90.65 \%$ for Q-LCDT). However, V/Q SPECT was significantly more sensitive (V/Q SPECT, 79.21\%; V/Q planar imaging, 75.84\% [P = 0.012]; Q-LDCT, $74.91 \%$ $[P<0.001])$, and $\mathrm{V} / \mathrm{Q}$ planar imaging was significantly more specific (V/Q planar imaging, 54.14\%; V/Q SPECT, 46.05\% [P $<0.001$ ]; QLDCT, $46.05 \%[P=0.001])$ than the other 2 techniques for identifying perfusion defects in individual lung segments. Conclusion: Both V/Q planar imaging and V/Q SPECT were highly effective for diagnosing CTEPH, and Q-LDCT may be a reliable alternative method for patients who are unsuitable for ventilation imaging.
\end{abstract}

Key Words: pulmonary hypertension; chronic thromboembolic pulmonary hypertension; ventilation-perfusion scanning; low-dose CT

J Nucl Med 2020; 61:1832-1838

DOI: 10.2967/jnumed.120.243188

Received Feb. 4, 2020; revision accepted Mar. 16, 2020.

For correspondence or reprints contact: Wei Fang, Fuwai Hospital, Chinese Academy of Medical Sciences, 167 N. Lishi Rd., Xicheng District, Beijing, 100037 China.

E-mail: nuclearfw@126.com

${ }^{*}$ Contributed equally to this work.

Published online Mar. 20, 2020.

COPYRIGHT (C 2020 by the Society of Nuclear Medicine and Molecular Imaging.
$\mathbf{C}$ hronic thromboembolic pulmonary hypertension (CTEPH) is classified as group 4 pulmonary hypertension $(\mathrm{PH})$ in guidelines published by the European Society of Cardiology and European Respiratory Society (1). It is characterized by the presence of macroscopic thromboembolic lesions in the proximal or distal pulmonary arteries and microscopic pulmonary vasculopathy (2), which impede blood flow and increase pressure in the pulmonary arteries. The lesions also produce lung regions that are adequately ventilated during inhalation but inadequately perfused by the pulmonary circulation, and these ventilation-perfusion (V/Q) mismatches can be detected, with high sensitivity and specificity, via imaging techniques that map both ventilation and perfusion in the lungs (3). Thus, V/Q scanning is the recommended procedure for identifying or excluding the presence of CTEPH during the diagnosis of PH (1).

Two primary methods of V/Q scanning are currently available: V/Q planar imaging, which was first introduced in the 1960s and produces 2-dimensional images, and V/Q SPECT, a more advanced, 3-dimensional technique. Of the two, V/Q SPECT is less likely to produce nondiagnostic scans and is more sensitive for diagnosing acute pulmonary embolism (PE) (4,5); however, the techniques have yet to be compared in patients with CTEPH. Furthermore, some evidence indicates that acute PE can also be diagnosed by combining a perfusion scan with a chest radiograph or thoracic CT scan (6), suggesting that ventilation scanning may be unnecessary for the diagnosis of CTEPH. Thus, the study described in this report was designed to prospectively compare the diagnostic performance of V/Q planar imaging, V/Q SPECT, and a combination of perfusion SPECT with low-dose CT (Q-LDCT) in patients with CTEPH. Pulmonary angiography assessments, which were once considered the gold standard for PE diagnosis, were also conducted to serve as a single, unambiguous benchmark for calculations of sensitivity, specificity, and accuracy.

\section{MATERIALS AND METHODS}

\section{Study Design and Population}

This study complied with the amended Declaration of Helsinki and was approved by the Institutional Review Board of Fuwai Hospital (ethical approval 402); all participants provided written informed consent. Patients referred to the National Center for Cardiovascular Disease and Fuwai Hospital for suspected PH were recruited prospectively from February 2016 to September 2018 and diagnosed according to the algorithm published in the guidelines on diagnosis and treatment of PH by the European Society of Cardiology and European Respiratory 
Society (Fig. 1) (1). Patients diagnosed with PH attributable to left heart disease or lung disease were excluded before the V/Q scanning procedure was attempted; additional exclusion criteria included failure to complete right heart catheterization or pulmonary angiography, and a resting mean pulmonary arterial pressure of less than $25 \mathrm{~mm}$ $\mathrm{Hg}$ on catheterization. Patients whose $\mathrm{PH}$ diagnosis was confirmed via right heart catheterization and who completed the V/Q planar imaging, V/Q SPECT, Q-LDCT, and pulmonary angiography procedures were included in the study. Our previous experience at the center indicated that approximately $35 \%$ of recruited patients would be diagnosed with CTEPH via our benchmark assessment (pulmonary angiography); thus, the planned enrollment was about 200 patients, yielding populations of about 70 patients in the CTEPH group and about 130 patients in the non-CTEPH group. On the basis of the anticipated sensitivity (95\%) and specificity (90\%) scores, with a $2-$ sided significance $(\alpha)$ of 0.05 , these sample sizes would estimate the performance of each technique for diagnosing CTEPH in patients with a precision of less than $5 \%$ (half the width of the $95 \%$ confidence interval).

\section{V/Q Planar Imaging, V/Q SPECT, and Q-LDCT}

V/Q scanning and chest LDCT were performed with hybrid SPECT/CT cameras (SymbiaT16 SPECT/CT [Siemens]; Discovery NM640 SPECT/CT [GE Healthcare]). The imaging protocols were conducted over $2 \mathrm{~d}$ with a dual-head $\gamma$-camera equipped with lowenergy, high-resolution, parallel-hole collimators. On the first day, perfusion planar scintigraphy, perfusion SPECT, and chest LDCT were performed consecutively. For perfusion planar scintigraphy, the patients were supine and intravenously injected with $111-185 \mathrm{MBq}$ of ${ }^{99 \mathrm{~m}} \mathrm{Tc}$-macroaggregated albumin $\left(2-7 \times 10^{5}\right.$ particles $)$; then, perfusion planar imaging was performed with a matrix size of $256 \times 256$ in 8 views (anterior, posterior, left anterior oblique, left lateral, left posterior oblique, right anterior oblique, right lateral, and right posterior oblique), and a total of 500 kilocounts per projection were collected. Immediately after the planar acquisition, SPECT images were acquired with a matrix size of $64 \times 64$, a zoom of 1.0 , and $3^{\circ}$ per frame over $360^{\circ}$; the duration of each projection was $10 \mathrm{~s}$. Chest LDCT was performed without contrast enhancement and with the following parameters: pitch of 1.25 , rotation time of $1.0 \mathrm{~s}$, effective tube current-time product of $30 \mathrm{mAs}$, and tube voltage of $120 \mathrm{kV}$; filtered backprojection was used for CT reconstruction. CT images were reconstructed with commonly used parameters for slice thickness $(2.5 \mathrm{~mm})$

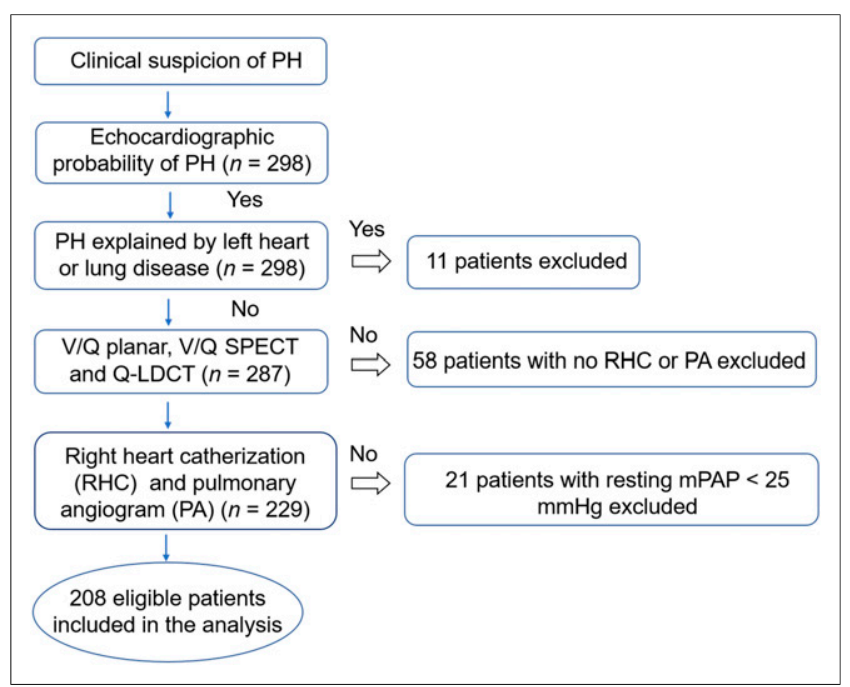

FIGURE 1. Patient-selection algorithm. and increment $(1.25 \mathrm{~mm})$. On the second day, ventilation planar scintigraphy and ventilation SPECT were performed. Patients inhaled 20$30 \mathrm{MBq}$ of ${ }^{99 \mathrm{~m} T c-T e c h n e g a s ~(C y c l o m e d i c a) ; ~ t h e n, ~ v e n t i l a t i o n ~ p l a n a r ~}$ scanning was conducted in the same manner as perfusion planar scanning, and ventilation SPECT images were acquired immediately afterward with a zoom of 1.0 and $3^{\circ}$ per frame over $360^{\circ}$. The duration of each projection was $15 \mathrm{~s}$. Reconstruction for both perfusion and ventilation SPECT was performed via ordered-subset expectation maximization with 8 subsets and 2 iterations. The estimated effective radiation dose from V/Q scanning was $2.1 \mathrm{mSv}$, the average doselength product for chest LDCT was $153.5 \mathrm{mGy} \times \mathrm{cm}$, and the average measured effective dose was $2.1 \pm 0.62 \mathrm{mSv}$. The total effective dose (V/Q + LDCT) was $4.2 \mathrm{mSv}$.

\section{Image Interpretation}

V/Q and LDCT images were interpreted by 2 experienced nuclear physicians who were masked to the clinical results, and the diagnosis was determined via consensus reading. To avoid recall bias, V/Q planar images were reviewed first, V/Q SPECT images were reviewed 1 wk later, and the LDCT and fused Q-LDCT images were reviewed $1 \mathrm{wk}$ after the V/Q SPECT images. V/Q images from both planar imaging and SPECT were interpreted - and a diagnosis of PE determined-according to the 2009 European Association of Nuclear Medicine guidelines for V/Q scintigraphy (7); the guidelines do not differentiate between acute PE and CTEPH; consequently, any perfusion defect meeting the criteria is classified as PE. A V/Q mismatch in at least 1 segment or 2 subsegments that conformed with the pulmonary vascular anatomy was considered diagnostic for PE, whereas multiple V/Q abnormalities that were not typical for specific diseases were considered nondiagnostic for PE. Criteria for the absence of PE were a normal perfusion pattern conforming to the anatomic boundaries of the lungs; in the absence of mismatch, the criteria for the absence of PE were matched or reversed-mismatch V/Q defects of any size, shape, or number or a mismatch that did not have a lobar, segmental, or subsegmental pattern. LDCT and fused Q-LDCT images in axial, coronal, and sagittal planes were displayed for review using 3-dimensional registration software (Xeleris 3 Functional Imaging Workstation; GE Healthcare). LDCT and Q-SPECT images were reviewed separately, and any perfusion defect in the absence of a CT abnormality was identified as a mismatch; mismatches in at least 1 segment or 2 subsegments from Q-LDCT imaging were considered diagnostic for PE (Figs. 2A and 2B).

\section{Pulmonary Angiography and Pulmonary Hemodynamic Measurements}

Pulmonary angiography was performed with an Allura Xper FD10/ 10 angiographic apparatus (Philips); 25 images were obtained per second with a matrix size of $1,024 \times 1,024$, and all images were collected with the same imaging parameters. The patient's right or left common femoral vein was cannulated, and a 6-French sheath (Cordis) was introduced. For angiograms of the main pulmonary arteries, a power injector was used to deliver $30-40 \mathrm{~mL}$ of iohexol (Omnipaque 350; GE Healthcare) through a 6-French pigtail catheter (Cordis) at a rate of $15-20 \mathrm{~mL} / \mathrm{s}$. For subselective studies, a hand or power injector was used to deliver $10-15 \mathrm{~mL}$ of the contrast material through a 5-French curved-tip catheter (Cordis) at approximately 5-8 $\mathrm{mL} / \mathrm{s}$. Anterior and supplemental oblique projections were obtained, and all images were evaluated by 2 experienced physicians.

Hemodynamic parameters (mean right atrial pressure, mean pulmonary arterial pressure, and pulmonary artery wedge pressure) were recorded during right heart catheterization within $1 \mathrm{wk}$ after V/Q scanning. Cardiac output was calculated as the mean value of 3 measurements obtained via the thermodilution method, and pulmonary vascular resistance and total pulmonary resistance were calculated: pulmonary 

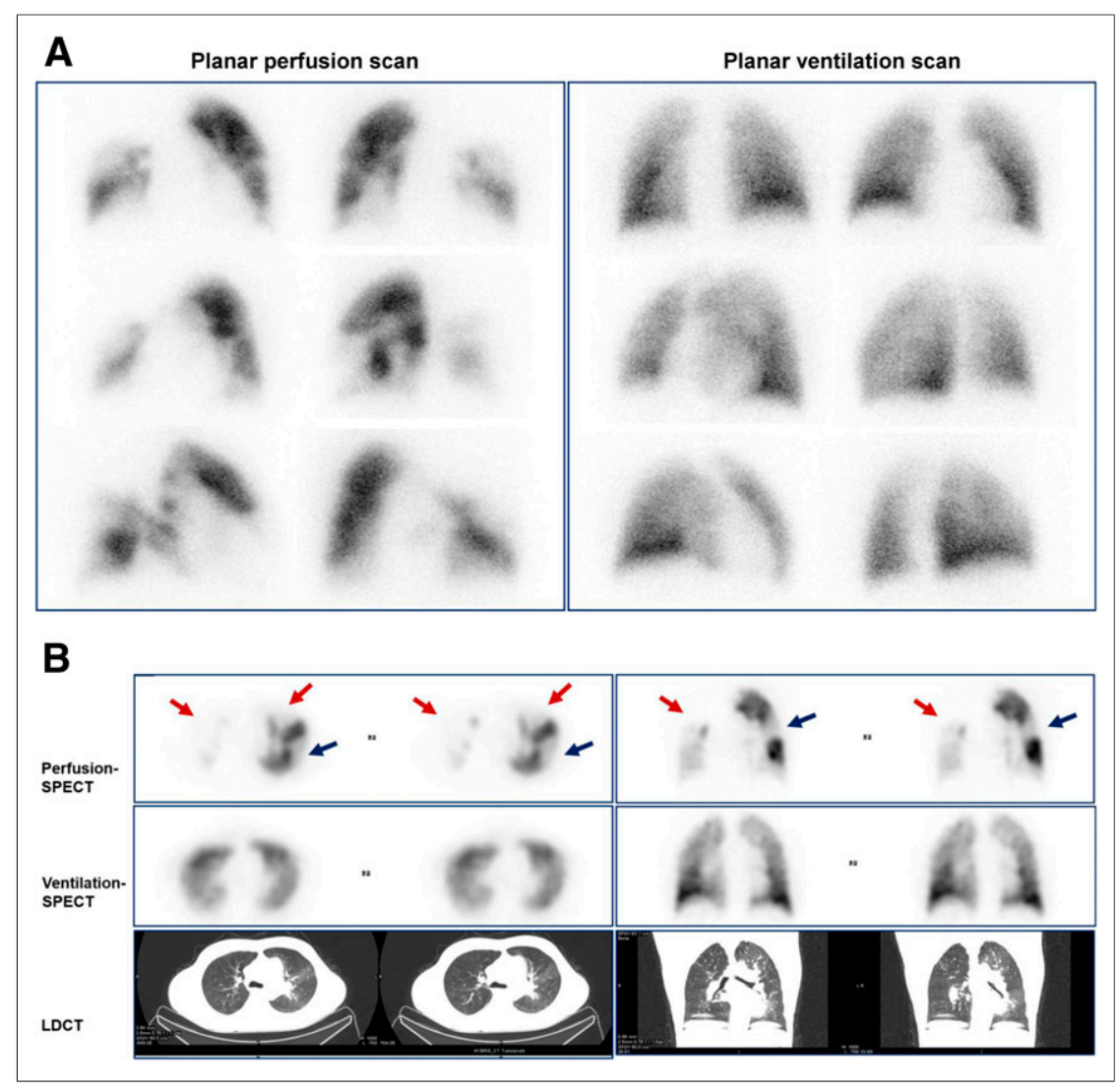

FIGURE 2. Representative images from CTEPH patient. (A) V/Q planar images show multiple segmental and subsegmental mismatched perfusion defects throughout right lung and in anterior segment, lingular segments, and basal segments of left lung. (B) Consistent with findings from V/Q planar imaging, V/Q SPECT and Q-LDCT images reveal mismatched perfusion defects in right lung and anterior segment of left lung (red arrows); however, posterior segment of left lung (blue arrows) displays mismatched perfusion defect in V/Q SPECT and Q-LDCT images but not in V/Q planar images.

vascular resistance $=($ mean pulmonary arterial pressure - pulmonary artery wedge pressure)/cardiac output; total pulmonary resistance $=$ mean pulmonary arterial pressure/cardiac output.

\section{Statistical Analysis}

Continuous data were expressed as mean $\pm \mathrm{SD}$, and categoric data were expressed as frequency and percentage. Differences between the 2 groups were analyzed for significance with the unpaired Student $t$ test for continuous variables and with the Fisher exact test for categoric variables. Pulmonary angiography was used as a benchmark for evaluating the diagnostic performance of each method. Differences in sensitivity, specificity, and accuracy were analyzed with the McNemar test, and the $\mathrm{\kappa}$-value was estimated to determine the degree of agreement between each method and pulmonary angiography. All statistical analyses were performed with SPSS software (version 19.0; SPSS Inc.), and significance was defined as a 2-tailed $P$ value of less than 0.05 .

\section{RESULTS}

\section{Patient Baseline Characteristics}

Ninety patients were excluded from the study: 11 with $\mathrm{PH}$ attributable to left heart disease or lung disease, 58 who failed to complete the right heart catheterization or pulmonary angiography procedures, and 21 whose resting mean pulmonary arterial pressure was less than $25 \mathrm{~mm} \mathrm{Hg}$ on right heart catheterization. In total, 208 patients were included in the study (Table 1), 69 of whom (33.2\%) were diagnosed with CTEPH when evaluated via pulmonary angiography. There were no nondiagnostic decisions during V/Q interpretation. Of the 139 patients with nonCTEPH diagnoses, 135 were diagnosed with group 1 PH (idiopathic pulmonary arterial hypertension, 69; heritable pulmonary arterial hypertension, 3; pulmonary arterial hypertension associated with connective tissue disease, 27; congenital heart disease, 35; pulmonary veno-occlusive disease, 1 ) and 4 were diagnosed with group $5 \mathrm{PH}(\mathrm{PH}$ associated with fibrosing mediastinitis, 1; PH of unclear mechanisms, 3).

\section{Diagnostic Performance of V/Q Planar Imaging and V/Q SPECT}

When pulmonary angiography was used as the benchmark for CTEPH diagnosis, V/Q planar imaging had a sensitivity of $94.20 \%$ and a specificity of $92.81 \%$, whereas V/Q SPECT had a sensitivity of $97.10 \%$ and a specificity of $91.37 \%$. Neither sensitivity nor specificity differed significantly between V/Q planar imaging and V/Q SPECT. Calculations of accuracy, as well as positive and negative predictive values, were also similar between techniques (accuracy: V/Q planar imaging, 93.27\%, and V/Q SPECT, 93.27\%; positive predictive value: V/Q planar imaging, $86.67 \%$, and V/Q SPECT, $84.81 \%$; negative predictive value: V/Q planar imaging, 96.99\%, and V/Q SPECT, $98.45 \%$ ) (Table 2), whereas $\kappa-$ analyses indicated that the results from both techniques agreed well with each other $(\kappa=0.897)$ and with the results from pulmonary angiography (V/Q planar imaging vs. pulmonary angiography: $\kappa=0.851$; V/Q SPECT vs. pulmonary angiography: $\kappa=$ 0.854).

The effectiveness of V/Q planar imaging and V/Q SPECT for identifying perfusion defects was also evaluated segmentally for the 69 patients whose CTEPH diagnoses were confirmed via pulmonary angiography. Assessments were based on a 20-segment lung model, and the results obtained from the interpretation of V/Q planar images and V/Q SPECT images for each individual segment were compared with the results of benchmark assessments (pulmonary angiography) in the same segment. In total, 1,380 lung segments were evaluated. An average of $12.91 \pm 4.9$ segments per patient displayed a mismatched pattern on V/Q planar imaging, whereas V/Q SPECT detected $13.94 \pm 4.8$ mismatched segments per patient. In total, 861 segments were positive for PE when evaluated via pulmonary angiography, compared with 891 and 962 that displayed mismatches when evaluated via V/Q planar imaging and V/Q SPECT, respectively. Thus, mismatched segments were more frequently identified via V/Q SPECT than via V/Q planar imaging, and this increase was accompanied by a correspondingly higher sensitivity (V/Q planar imaging, 75.84\%; V/Q SPECT, 79.21\% [P=0.012]) (Table 3). 
TABLE 1

Baseline Patient Characteristics

\begin{tabular}{|c|c|c|c|}
\hline Characteristic & All patients $(n=208)$ & CTEPH $(n=69)$ & Non-CTEPH $(n=139)$ \\
\hline Age (y) & $42.0 \pm 15.6$ & $54.1 \pm 13.1$ & $36.1 \pm 13.2$ \\
\hline Female $(n)$ & $140(67.3 \%)$ & $37(53.6 \%)$ & $103(74.1 \%)$ \\
\hline \multicolumn{4}{|l|}{ NYHA class $(n)$} \\
\hline 1 & 9 & 0 & 9 \\
\hline ॥ & 94 & 29 & 65 \\
\hline III & 97 & 39 & 58 \\
\hline IV & 8 & 1 & 7 \\
\hline \multicolumn{4}{|l|}{$\mathrm{RHC}$} \\
\hline mRAP $(\mathrm{mm} \mathrm{Hg})$ & $6.8 \pm 5.3$ & $7.7 \pm 6.3$ & $6.4 \pm 4.7$ \\
\hline mPAP (mm Hg) & $53.2 \pm 15.9$ & $48.3 \pm 11.4$ & $55.6 \pm 17.3$ \\
\hline mPAWP (mm Hg) & $8.9 \pm 4.1$ & $10.2 \pm 4.3$ & $8.3 \pm 3.8$ \\
\hline $\mathrm{Cl}\left(\mathrm{L} / \mathrm{min} / \mathrm{m}^{2}\right)$ & $2.8 \pm 0.8$ & $2.7 \pm 0.7$ & $2.9 \pm 0.9$ \\
\hline PVR (Wood units) & $10.8 \pm 6.2$ & $9.3 \pm 4.5$ & $11.8 \pm 7.0$ \\
\hline TPR $\left(\right.$ dyn $\left.\times \mathrm{s} \times \mathrm{cm}^{2}\right)$ & $1,037.6 \pm 502.3$ & $930.0 \pm 393.9$ & $1,087.0 \pm 539.0$ \\
\hline \multicolumn{4}{|l|}{ Blood test } \\
\hline Big ET-1 (pmol/L) & $0.5 \pm 0.6$ & $0.6 \pm 0.7$ & $0.5 \pm 0.5$ \\
\hline NT-proBNP (pg/mL) & $1,286.0 \pm 1,816.0$ & $1,492.2 \pm 1,735.7$ & $1,183.6 \pm 1,851.7$ \\
\hline D-dimer (ng/mL) & $618.7 \pm 1,677.0$ & $927.5 \pm 2,478.7$ & $465.4 \pm 1,058.1$ \\
\hline 6-min WD (m) & $421.4 \pm 46.1$ & $371.7 \pm 74.6$ & $437.4 \pm 95.8$ \\
\hline
\end{tabular}

$\mathrm{NYHA}=$ New York Heart Association; RHC = right heart catherization; mRAP = mean right atrial pressure; $\mathrm{mPAP}=$ mean right pulmonary artery pressure; mPAWP $=$ mean pulmonary artery wedge pressure; $\mathrm{Cl}=$ cardiac index; PVR $=$ pulmonary vascular resistance; TPR = total pulmonary resistance; ET-1 = endothelin-1; NT-proBNP = N-terminal pro b-type natriuretic peptide; WD = walking distance.

Qualitative data are numbers followed by percentages in parentheses; continuous data are mean \pm SD.

However, specificity was significantly lower for V/Q SPECT than for V/Q planar imaging (V/Q planar imaging, 54.14\%; V/Q SPECT, $46.05 \%[P<0.001])$, whereas the 2 techniques were similar in accuracy (V/Q planar imaging, 67.68\%; V/Q SPECT, $66.74 \%$ ), positive predictive value (V/Q planar imaging, $73.29 \%$; V/Q SPECT, 70.89\%), and negative predictive value (V/Q planar imaging, 57.46\%; V/Q SPECT, 57.18\%). Thus, although mismatched segments were more likely to be correctly identified via V/Q SPECT than via V/Q planar imaging, V/Q SPECT also led to a greater number of false CTEPH indicators, and the overall accuracy of the 2 techniques was similar.

\section{Diagnostic Performance of Q-LDCT}

When patients were evaluated via Q-LDCT, 79 were positive and 129 were negative for CTEPH. Sixty-six of the CTEPHpositive and 126 of the CTEPH-negative patients were diagnosed with and without CTEPH, respectively, via pulmonary angiography, and calculations of sensitivity (95.65\%), specificity $(90.65 \%)$, accuracy $(92.31 \%)$, positive predictive value $(83.54 \%)$, and negative predictive value $(97.67 \%)$ for the Q-LDCT analyses of individual patients did not differ significantly from the corresponding calculations for V/Q planar imaging or V/Q SPECT (Table 2). For segmental analyses, defects were identified as mismatches between perfusion (Q-SPECT) and CT images in at least 1 segment or 2 subsegments, and our results indicated that QLDCT was significantly less sensitive $(74.91 \% ; P<0.001)$ than
V/Q SPECT, significantly less specific $(46.05 \% ; P=0.001)$ than V/Q planar imaging, and significantly less accurate $(64.06 \% ; P=$ 0.005 vs. V/Q planar, $P=0.003$ vs. V/Q SPECT) than either of the other 2 techniques (Table 3 ).

\section{DISCUSSION}

V/Q scanning was first established in the 1960s and, after more than a half-century of development, has become the first-line imaging technique for diagnosing $\mathrm{PE}$ because of its noninvasiveness, low radiation burden, and high sensitivity. The joint task force of the European Society of Cardiology and the European Respiratory Society recommends that V/Q scanning be used to identify or exclude CTEPH during an early stage of the algorithm for diagnosing $\mathrm{PH}$; the report of the task force suggests that V/Q SPECT may be more effective than V/Q planar imaging (1), but the 2 techniques have yet to be rigorously compared. Thus, this investigation was the first, to our knowledge, to prospectively evaluate the effectiveness of V/Q planar imaging and V/Q SPECT for CTEPH diagnosis in a large group of patients with $\mathrm{PH}$, and our results confirmed that both techniques were highly effective for detecting or excluding CTEPH in individual patients, with no significant differences in sensitivity, specificity, or accuracy. Our results also indicate that Q-LDCT could be a reliable alternative method for identifying CTEPH in patients with $\mathrm{PH}$ when ventilation methods are unavailable. 
V/Q SPECT is considered more accurate than V/Q planar imaging for evaluations of acute PE by an increasing majority of physicians and researchers (8). However, previous investigations of the diagnostic performance of lung-scanning technology for CTEPH have yielded varying results $(9,10)$, and extrapolating the findings from studies of acute PE to CTEPH may be problematic, especially since the benchmark parameter for many of the acute PE studies was generated from a composite of measures, such as clinical symptoms, laboratory tests, observations at follow-up, and, sometimes, V/Q planar imaging or V/Q SPECT. Pulmonary angiography is rarely performed now, because CT angiography is less invasive and has similar diagnostic accuracy (11). Nevertheless, conventional x-ray pulmonary angiography is often required to identify patients with CTEPH who may benefit from pulmonary endarterectomy or pulmonary balloon angioplasty and can be performed during right heart catheterization (12). Thus, pulmonary angiography was chosen as the benchmark for the studies reported here because it is unambiguous, reliable, and clinically relevant for studies of CTEPH.

Although both acute PE and CTEPH are caused by obstruction of pulmonary arteries, their underlying pathologies differ substantially. For example, the pulmonary artery obstructions in patients with CTEPH are more diffuse and multisegmental, as demonstrated by our observation that the patients with CTEPH in this study typically displayed V/Q mismatches in the majority $(\sim 13 / 20)$ of lung segments. Obstructions in a single small segment or subsegment are also very unlikely to cause CTEPH but are not uncommon in patients with acute PE. Furthermore, $\mathrm{PH}$ is a fundamental component of the CTEPH diagnosis and is characterized by remodeling of pulmonary vessels, including narrowing or distortion of distal pulmonary arteries, which can lead to microvasculature embolism but no evidence of thrombosis during anatomic imaging (even pulmonary angiography). For these reasons, the apparent superiority of V/Q SPECT to V/Q planar imaging for diagnosing acute $\mathrm{PE}$ may not be translatable to patients with CTEPH. Notably, PH itself can also lead to perfusion defects that are detectable via V/Q planar imaging or V/Q SPECT $(13,14)$ - a fact that likely explains, at least in part, the occurrence of false-positive cases in our study.

The results from our investigation indicated that V/Q SPECT was more sensitive than V/Q planar imaging for identifying mismatches in individual segments or subsegments, as is consistent with the results from previous reports (15-17). Notably, the results from a pilot study by Soler et al. also indicated that perfusion SPECT was more sensitive than V/Q planar imaging for detecting segmental pulmonary artery obstructions in patients with $\mathrm{CTEPH}$; however, the ventilation scans were conducted with ${ }^{133} \mathrm{Xe}$ gas and performed only in the planar mode, because SPECT ventilation was not available (18). In principle, this increased sensitivity may lead to unnecessary diagnoses and overtreatment of clinically insignificant defects in patients with acute PE (19), but this concern is less relevant in patients with CTEPH, for whom lifelong anticoagulant therapy is recommended. Perhaps more importantly, these observations suggest that V/Q SPECT may be superior to V/Q planar imaging for identifying vessels that can be targeted during pulmonary endarterectomy and pulmonary balloon angioplasty $(20,21)$, which are the most promising treatments for improving symptoms and prognoses in eligible patients with CTEPH (12). Thus, although our results suggested that V/Q SPECT is no more effective than V/Q planar imaging for diagnosing CTEPH in individual patients, the greater sensitivity of V/Q 
SPECT may be advantageous for certain therapeutic approaches and for postoperative assessments of newly acute PE (22).

Despite detailed guidelines recommending that V/Q scanning be conducted for all patients in whom $\mathrm{PH}$ is suspected, only $57 \%$ of patients registered in the Pulmonary Arterial HypertensionQuality Enhancement Research Initiative underwent V/Q scanning (23). This discrepancy may be partially attributable to the broad availability of more routine clinical procedures, such as chest radiography or CT, and as SPECT/CT hybrid imaging systems become more common, the popularity of Q-LDCT-based diagnostic techniques may increase accordingly. Recent attempts to measure the accuracy of Q-LDCT have been somewhat inconsistent (24-26), chiefly because specificity estimates have ranged from $94 \%$ to as low as $51 \%$, but the implications of these investigations (like those comparing the effectiveness of V/Q planar imaging and V/Q SPECT) are difficult to interpret for CTEPH diagnoses because they were conducted retrospectively on patients with PE and are confounded by the use of composite benchmarks. Thus, our observation (from a prospective study with an unambiguous and clinically relevant benchmark) that CTEPH can be effectively diagnosed via Q-LDCT could have a substantial impact on patient care, particularly for individuals whose clinical instability or symptoms (e.g., severe dyspnea) preclude ventilation imaging. Q-LDCT examinations also require less time than V/Q scanning and could provide information about other thoracic pathologies (e.g., pneumonia, bullous emphysema, and tumors) that may lead to perfusion abnormalities; however, the results from our segmental analyses suggest that the technique may be less accurate than either V/Q SPECT or V/Q planar imaging for identifying the precise location of perfusion defects. The concurrent presence of parenchymal conditions in patients with CTEPH can be depicted on CT images (e.g., ground-glass opacification), resulting in a matched Q-LDCT pattern, which led to the diagnosis of absence of PE. This condition resulted in more false-negative results for Q-LDCT, potentially lowering its sensitivity.

Because this study was conducted at a single national referral center for cardiovascular disease and $\mathrm{PH}$, the calculated positive predictive values and negative predictive values will be valid only for patient populations in which the incidence of CTEPH is high. Our study is also limited by sample size, which may not be sufficient to exclude differences in the performance of diagnostic modalities, and by the inclusion of patients with pulmonary arteritis, which may impact our specificity measurements. Arteritis assessments are not recommended in the algorithm of the joint task force of the European Society of Cardiology and European Respiratory Society, and V/Q imaging alone typically cannot differentiate between arteritis and PE, but the condition can be identified via clinical observation. Nevertheless, because only 9 arteritis cases were included in our study, they are unlikely to have significantly influenced our results. Our study was also restricted to the diagnostic performance of V/Q imaging; future studies are needed to determine whether the use of V/Q imaging can be expanded to include therapeutic applications, such as pulmonary endarterectomy and pulmonary balloon angioplasty (27).

\section{CONCLUSION}

The results from this investigation indicate that both V/Q planar imaging and V/Q SPECT were highly effective for detecting or excluding CTEPH in patients with $\mathrm{PH}$, with no significant differences in sensitivity, specificity, or accuracy observed between the 2 
techniques. Q-LDCT may be a reliable alternative method for identifying CTEPH in patients who are unsuitable for ventilation imaging; however, V/Q SPECT appeared to be superior to both methods for identifying perfusion defects in individual lung segments.

\section{DISCLOSURE}

This work was supported by grant 81801733 from National Natural Science Foundation of China. Lei Wang is supported by the Young Elite Scientists Sponsorship program of CAST (2018QNRC001). No other potential conflict of interest relevant to this article was reported.

\section{ACKNOWLEDGMENT}

We thank Dr. Yang Wang (Medical Research and Biometrics Center, Cardiovascular Institute and Fuwai Hospital, National Center for Cardiovascular Diseases) for his advice on statistical analysis.

\section{KEY POINTS}

QUESTION: Is the diagnostic performance of V/Q planar imaging, V/Q SPECT, and Q-LDCT comparable in patients with CTEPH?

PERTINENT FINDINGS: This prospective study showed that V/Q planar imaging and V/Q SPECT were highly effective for diagnosing CTEPH, and V/Q SPECT was more sensitive for identifying mismatches segmentally.

IMPLICATIONS FOR PATIENT CARE: Q-LDCT may be a reliable alternative method for patients who are unsuitable for ventilation imaging.

\section{REFERENCES}

1. Galiè N, Humbert M, Vachiery JL, et al. 2015 ESC/ERS guidelines for the diagnosis and treatment of pulmonary hypertension. The Joint Task Force for the Diagnosis and Treatment of Pulmonary Hypertension of the European Society of Cardiology (ESC) and the European Respiratory Society (ERS). Eur Respir J. 2015;46:903-975.

2. Simonneau G, Torbicki A, Dorfmüller P, et al. The pathophysiology of chronic thromboembolic pulmonary hypertension. Eur Respir Rev. 2017;26:160111.

3. Tunariu N, Gibbs SJ, Win Z, et al. Ventilation-perfusion scintigraphy is more sensitive than multidetector CTPA in detecting chronic thromboembolic pulmonary disease as a treatable cause of pulmonary hypertension. J Nucl Med. 2007; 48:680-684

4. Gutte H, Mortensen J, Jensen CV, et al. Comparison of V/Q SPECT and planar V/Q lung scintigraphy in diagnosing acute pulmonary embolism. Nucl Med Commun. 2010;31:82-86.

5. Stubbs M, Chan K, McMeekin $\mathrm{H}$, et al. Incidence of a single subsegmental mismatched perfusion defect in single-photon emission computed tomography and planar ventilation/perfusion scans. Nucl Med Commun. 2017;38: $135-140$.
6. Stein PD, Terrin ML, Gottschalk A, et al. Value of ventilation/perfusion scans versus perfusion scans alone in acute pulmonary embolism. Am J Cardiol. 1992; 69:1239-1241.

7. Bajc M, Neilly JB, Miniati M, et al. EANM guidelines for ventilation/perfusion scintigraphy: part 1. Pulmonary imaging with ventilation/perfusion single photon emission tomography. Eur J Nucl Med Mol Imaging. 2009;36:1356-1370.

8. Stein PD, Freeman LM, Sostman HD, et al. SPECT in acute pulmonary embolism. J Nucl Med. 2009;50:1999-2007.

9. Worsley DF, Palevsky HI, Alavi A. Ventilation-perfusion lung scanning in the evaluation of pulmonary hypertension. J Nucl Med. 1994;35:793-796.

10. Johns CS, Swift AJ, Rajaram S, et al. Lung perfusion: MRI vs. SPECT for screening in suspected chronic thromboembolic pulmonary hypertension. J Magn Reson Imaging. 2017;46:1693-1697.

11. van Beek EJ, Reekers JA, Batchelor DA, et al. Feasibility, safety and clinical utility of angiography in patients with suspected pulmonary embolism. Eur Radiol. 1996;6:415-419.

12. Jenkins D, Madani M, Fadel E, et al. Pulmonary endarterectomy in the management of chronic thromboembolic pulmonary hypertension. Eur Respir Rev. 2017;26: 160111.

13. Wang M, Ma R, Wu D, et al. Value of lung perfusion scintigraphy in patients with idiopathic pulmonary arterial hypertension: a patchy pattern to consider. Pulm Circ. 2019;9:2045894018816968.

14. Chan K, Ioannidis S, Coghlan JG, et al. Pulmonary arterial hypertension with abnormal V/Q single-photon emission computed tomography. JACC Cardiovasc Imaging. 2018;11:1487-1493.

15. Bajc M, Olsson CG, Olsson B, et al. Diagnostic evaluation of planar and tomographic ventilation/perfusion lung images in patients with suspected pulmonary emboli. Clin Physiol Funct Imaging. 2004;24:249-256.

16. Reinartz P, Wildberger JE, Schaefer W, et al. Tomographic imaging in the diagnosis of pulmonary embolism: a comparison between V/Q lung scintigraphy in SPECT technique and multislice spiral CT. J Nucl Med. 2004;45:1501-1508.

17. Collart JP, Roelants V, Vanpee D, et al. Is a lung perfusion scan obtained by using single photon emission computed tomography able to improve the radionuclide diagnosis of pulmonary embolism? Nucl Med Commun. 2002;23:1107-1113.

18. Soler X, Hoh CK, Test VJ, et al. Single photon emission computed tomography in chronic thromboembolic pulmonary hypertension. Respirology. 2011;16:131137.

19. Metter D, Tulchinsky M, Freeman LM. Current status of ventilation-perfusion scintigraphy for suspected pulmonary embolism. AJR. 2017;208:489-494.

20. Kawakami T, Kataoka M, Nakahara T, et al. Usefulness of 3D SPECT/CT fusion image in CTEPH. Int J Cardiol. 2015;194:39-40.

21. Hosokawa K, Abe K, Kashihara S, et al. 3-dimensional SPECT/CT fusion imaging-guided balloon pulmonary angioplasty for chronic thromboembolic pulmonary hypertension. JACC Cardiovasc Interv. 2017;10:e193-e194.

22. Moradi F, Morris TA, Hoh CK. Perfusion scintigraphy in diagnosis and management of thromboembolic pulmonary hypertension. Radiographics. 2019;39:169185 .

23. McLaughlin VV, Langer A, Tan M, et al. Contemporary trends in the diagnosis and management of pulmonary arterial hypertension: an initiative to close the care gap. Chest. 2013;143:324-332.

24. Gutte H, Mortensen J, Jensen CV, et al. Detection of pulmonary embolism with combined ventilation-perfusion SPECT and low-dose CT: head-to-head comparison with multidetector CT angiography. J Nucl Med. 2009;50:1987-1992.

25. Palmowski K, Oltmanns U, Kreuter M, et al. Diagnosis of pulmonary embolism: conventional ventilation/perfusion SPECT is superior to the combination of perfusion SPECT and nonenhanced CT. Respiration. 2014;88:291-297.

26. Lu Y, Lorenzoni A, Fox JJ, et al. Noncontrast perfusion single-photon emission CT/CT scanning: a new test for the expedited, high-accuracy diagnosis of acute pulmonary embolism. Chest. 2014;145:1079-1088.

27. Harris B, Bailey D, Miles S, et al. Objective analysis of tomographic ventilationperfusion scintigraphy in pulmonary embolism. Am J Respir Crit Care Med. 2007; 175:1173-1180. 